\title{
A Maximum Likelihood (ML) based OSTBC-OFDM System over Multipath Fading Channels
}

\author{
Ramaswamy $\mathrm{T}$. \\ Assistant Professor \\ MREC, Hyderabad
}

\author{
K. Chennakesava Reddy, PhD \\ Principal \\ BITSW, Hyderabad
}

\begin{abstract}
This paper proposes a space-time block-coded orthogonal frequency-division multiplexing (STBC-OFDM) scheme for frequency-selective fading channels which does not require channel knowledge either at the transmitter or at the receiver. This paper proposed a generalized maximum likelihood estimation decoding algorithm. Due to the orthogonality nature of STBC, the rule for decoding was reduced to a single step. The performance investigation of the proposed system was done over Rayleigh fading channel and also over the Rician fading channel. Simulation results reveal the performance of proposed STBC communication system is near optimum.
\end{abstract}

\section{Keywords}

MIMO, STBC-OFDM, Almouti Scheme, Maximum likelihood estimator, BER.

\section{INTRODUCTION}

The target of next generation wireless communication is to achieve high data rates with low bandwidth. It should be power efficient. At present time, Orthogonal Frequency Division Multiplexing is widely used for its bandwidth efficiency property because of its orthogonal characteristic and more data can be transmitted at a certain amount of bandwidth compare to the other systems. Orthogonal frequency division multiplexing (OFDM) is being considered to be the most promising multiplexing techniques to support the future wireless multimedia communication system. It is because of its bandwidth efficiency performance. The affects of Inter Symbol interference (ISI) is also very less than the system compare to the other multiplexing techniques. OFDM has been adopted and implemented in wire and wireless communication system. Severe attenuation in a multipath wireless environment makes it extremely difficult for the receiver to determine the transmitted signal unless the receiver is provided with some form of diversity i.e. some lessattenuated replica of the transmitted signal is provided to the receiver. In some applications, the only practical means of achieving diversity is deployment of antenna array at the transmitter and/or at the receiver end. In recent days, the communication systems demands for highly bandwidthefficient and also highly power-efficient techniques are very valued for wireless communication in next generation systems. Compared to single antenna systems the multiple antenna systems offer an increased capacity of data rates. STBC was a bandwidth-efficient and also a power-efficient technique for communication through fading channel by using multiple transmitting antennas systems. Diversity gain enhancement is another technique for good performance achievement. Through MIMO-Space Time Block Code (STBC), the antenna diversity gain can be increased. This paper studies the performance of STBC-OFDM in the MIMO communication system under various channel constraints,
Rayleigh fading channel, Gaussian channel, etc. This paper also gives the details about the effect of modulation, power conditions, antenna selection techniques, fast and slow fading channel conditions. The remaining part of paper is ordered as follows: Section 2 gives the details about the system mode of STBC-OFDM. The decoding algorithm used to decode the information at the receiver is given in Section 3. Section 4 demonstrates the results of the proposed STBC system and finally conclusions are provided in section 5 .

\section{SYSTEM MODEL}

The block diagram of STBC-OFDM is illustrated in Fig.1. The binary data stream is first modulated and mapped to a sequence of complex modulation symbols. The modulated sequence is then passed through a serial-to-parallel converter producing $L$ data streams of length $M$ in each transmit branch. An $M$-point Inverse Discrete Fourier Transform (IDFT) is performed on each serial data stream. IDFT gives the output as a block of M-time domain samples of a respective OFDM frame. To sidestep the effects of inter-symbol-interference, a cyclic prefix (CP) is added to output samples. Then, every OFDM frame is nurtured into a space-time block encoder. This paper has used the simple Almouti's scheme [2] for simplicity, even the proposed approach can be applicable to the space-time block code from the orthogonal family design [3]. The $l$ th frames of first and second transmitting antennas are represented by,

$$
\begin{aligned}
& \left(X_{l}^{1}(0), \ldots, X_{l}^{1}(M-1)\right. \\
& \left(X_{l}^{2}(0), \ldots, X_{l}^{2}(M-1)\right.
\end{aligned}
$$

For the subsequent OFDM frame interval, the OFDM frame can be represented as

$\left(X_{l}^{1 *}(0), \ldots, X_{l}^{1 *}(M-1)\right.$

This is transmitted from the second antenna and

$\left(-X_{l}^{2 *}(0), \ldots,-X_{l}^{2 *}(M-1)\right.$

Transmits from the first antenna according to the signaling structure of Alamouti's scheme, where (.)* denotes complex conjugation.

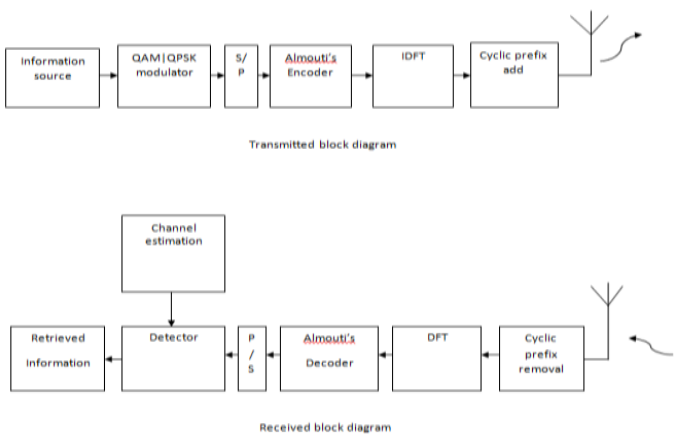

Fig.1 Overall system Model 
In the proposed system model, a FIR filter with the memory was used to represent channels belonging to the 2 transmit antennas. At the receiver, first the cyclic prefix is removed, and then the received signal is passed through a DFT operation. It can be shown that the OFDM demodulator output can be represented as,

$$
\begin{aligned}
& Y_{l}(k)=\sum_{m=1}^{2} X_{l}^{m}(k) H^{m}(k)+N_{l}(k), l= \\
& 1,2, \ldots, 2 L, \quad k=0 \ldots M-1
\end{aligned}
$$

Where $N_{l}(k)$ are independent samples of a Gaussian random variable with variance $N O$ and $H m(k)$ is the frequency response of the channel from the $m$ th transmit antenna to the single receive antenna at the $k$ th tone. After performing DFT operation, by considering the $k$ th frequency component of each and every frame, the receiver tries to construct a tone stream of $2 \mathrm{~L}$ frequency-domain samples. Such that, $2 \mathrm{~L}$ streams each of length $M$ are realigned onto $M$ streams, each of length $2 \mathrm{~L}$. The obtained stream was fed to the detecting algorithm and explained in the next section in detail. By considering the coding structure, let us define the following sub-matrices

$i=1,2 \ldots L$

$$
Y_{i}(k)=\left(\begin{array}{c}
Y_{2 i-1} \\
Y_{2 i}
\end{array}\right), \quad X_{i}(k)=\left[\begin{array}{cc}
X_{i}^{1}(k) & X_{i}^{2}(k) \\
-X_{i}^{2 *}(k) & X_{i}^{1 *}(k)
\end{array}\right]
$$

Then based on the above matrices and the system model the received signal receiver section can be written as

$Y(k)=X(k) H(k)+N(k)$

\section{DECODING ALGORITHM}

In this approach, the decoding algorithm is based on the generalized maximum likelihood estimation [9], just a twostep process. For every toned frequency $\mathrm{k}, \mathrm{k}=0,1, \ldots, \mathrm{M}-1$, the rules of decoding are to maximize and the respective likelihood logarithmic function is given as

$\ln p(Y(k) \mid X(k), H(k))=-\|Y(k)-X(K) H(k)\|^{2}$

Maximizing (8) with respect to $\boldsymbol{H}(k)$ yields the channel estimate (i.e. the $k$ th DFT coefficient of the channel impulse response),

$\widehat{H}(k)=\left(X^{\prime}(k) X(k)\right)^{-1} X^{\prime}(k) Y(k)$

In general for Almouti's STBC coding, space-time block codes are based on orthogonality conditions, the primary term in eq.(6) represents a scale identity matrix, thus the eq.(9) reduces to

$\widehat{H}(k)=X^{\prime}(k) Y(k)$

Introducing the channel estimate in eq.(5) produces the simple decoding rule

$\hat{X}(k)=\arg \max _{X(k)}\left\|X^{\prime}(k) Y(k)\right\|^{2}, k=0,1, \ldots, M-$ 1

Where $\hat{X}(k)$ consists of the estimates for the $k_{\mathrm{th}}$ component in each OFDM frame. By the collection of all the outputs of all $\mathrm{M}$ processors, the original signal can be reconstructed from both antennas. The decoding rule creates phase ambiguity at decoder. This ambiguity can be solved by setting the samples of decoder input to a fixed value. This procedure requires the starting frame of both antennas to be reconstructed with the help of pilot symbols.

\section{RESULTS}

This section gives the details about the performance evaluation of proposed approach. Simulations are done in MATLAB using the Rayleigh fading channel model. For each simulation, blocks of 1000 symbols are simulated. The proposed system was simulated with two transmitter antennas, two receiver antennas and also at maximum Doppler frequency of $50 \mathrm{~Hz} \quad(\mathrm{f}=50 \mathrm{~Hz})$. We have used channel conditions, which have two independent paths with path delays in seconds and average path gains $=\left[\begin{array}{ll}0 & -18\end{array}\right] \mathrm{dB}$. The simulation was done under various constraints like various types of modulation schemes, power allocation techniques and also over various fading channel assumptions. The respective figures are shown figure.2, figure. 3 and figure. 4 respectively. Table. 1 gives the details about the summarization of simulation parameters

\section{Case 1: STBC 2X2 MIMO with Rayleigh channel}

Table.1: Simulation Parameters

\begin{tabular}{|c|c|}
\hline Parameter & Value \\
\hline Number of Symbols & 1000 \\
\hline Number of Transmitters & 2 \\
\hline Number of Receivers & 2 \\
\hline Fading Effect & Rayleigh \\
\hline Noise & AWGN \\
\hline Doppler Frequency & $50 \mathrm{~Hz}$ \\
\hline SNR & $0-30$ \\
\hline System Model & MIMO \\
\hline
\end{tabular}

The order of the modulation stipulates the number of the diverse symbols that can be transmitted in a digital communication system. There are various modulation schemes like PSK, FSK and AM. In this paper, the proposed approach was tested over various PSK modulations like, BPSK, QPSK, 16-PSK and 32-PSK over Rayleigh fading channel and the respective results are illustrates in figure.2.

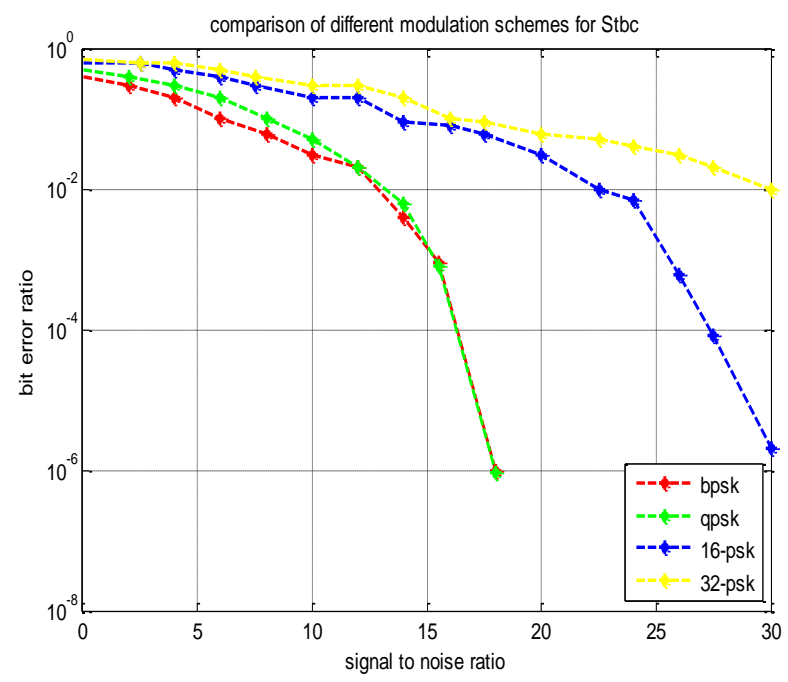

Fig.2. Performance comparison of $2 * 2$ STBC-OFDM with M-PSK Modulation

The above figure illustrates the details about the BER performance of proposed system with varying modulation technique for the same data stream. The above figure shows 
that there is a significant loss in the SNR with increasing in the modulation order and the proposed system has high BER. Here, one more comparison was illustrated though power allocation with respect to equal and non-equal powers allocated for TX antennas. In the case of equal power, both antennas having same power and in the case of unequal second antenna will receive less power compare to first antenna.

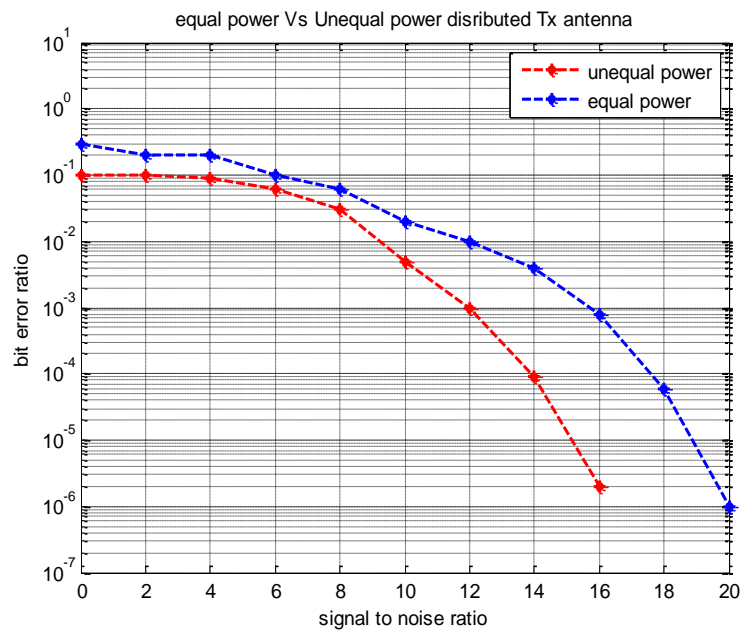

Fig.3 Performance comparison of $2 * 2$ STBC-OFDM with equal and unequal power distributions

From the fig. 3 , it can be noticed that equal power system gives a $3 \mathrm{~dB}$ penalty when compared with unequal power system. Fading is unconventionality of the attenuation that a carrier modulated telecommunication signal experiences over a particular propagation media. The terms, fast and slow fading demonstrates the rate at which the phase and magnitude of the signal was changing by the channel. The coherence time was the time above which the value of correlation function is above 0.5 . When the delay constraint of the channel was small compared to coherence time of channel, it is called slow fading channel. Fast fading channel can be defined as the channel at which the channel delay constraint if higher compared with coherence time of channel.

The Simulation results for different Doppler frequency (fd) $50,200,300,500$ are presented.

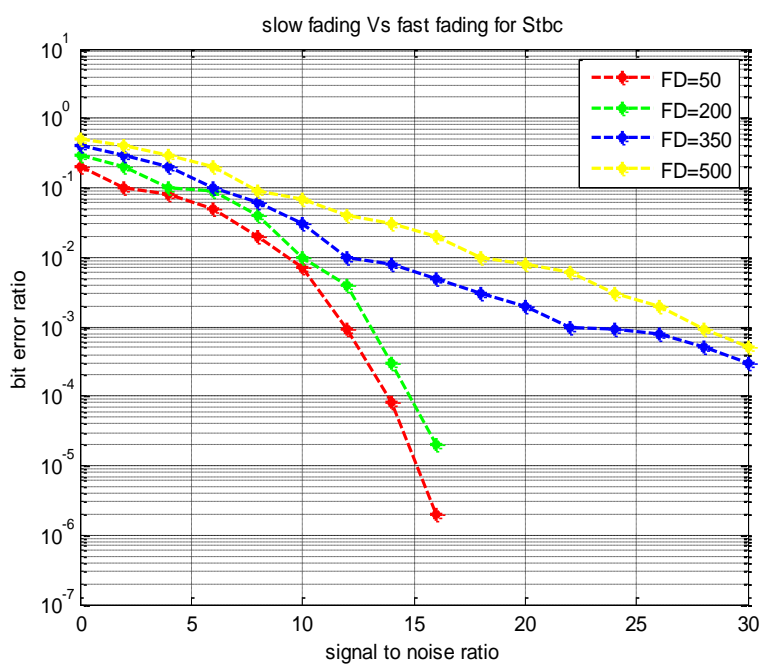

Fig.4. Performance comparison of $2 * 2$ STBC-OFDM at various Doppler frequencies
From the performance result in Figure 4, it can be seen that the performances of STBC-OFDM degrade in fast fading conditions.

Case 2: STBC 2X2 MIMO with Rician fading channel

Table.2: Simulation Parameters

\begin{tabular}{|c|c|}
\hline Parameter & Value \\
\hline Number of Symbols & 1000 \\
\hline Number of Transmitters & 2 \\
\hline Number of Receivers & 2 \\
\hline Fading Effect & Rician \\
\hline Noise & AWGN \\
\hline Doppler Frequency & $50 \mathrm{~Hz}$ \\
\hline SNR & $0-30$ \\
\hline System Model & MIMO \\
\hline
\end{tabular}

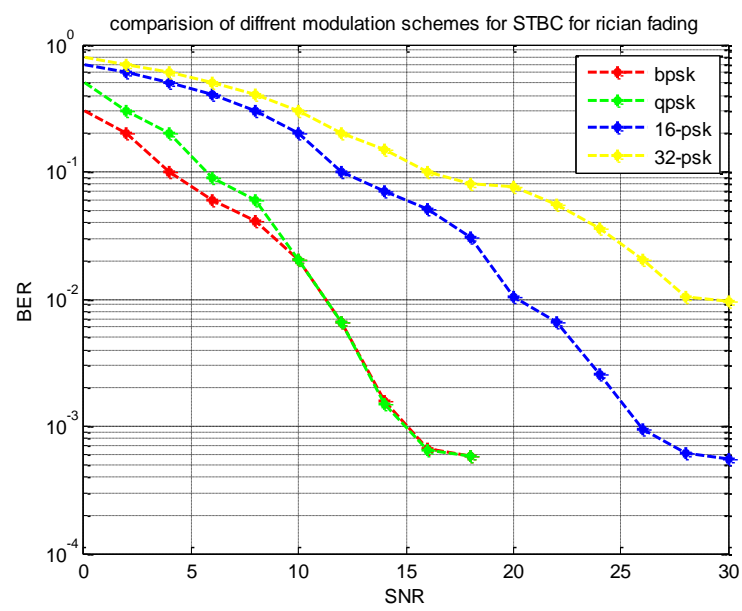

Fig.5. Performance comparison of $2 * 2$ STBC-OFDM with M-PSK Modulation

The modulation order specifies the number of the different symbols that can be transmitted by a digital communication system. The simulation results in figure. 5 show that increasing the order of modulation will result in a significant SNR loss over rician fading channel. Therefore, it can be said that for the higher order of modulation order, the system will have higher BER.

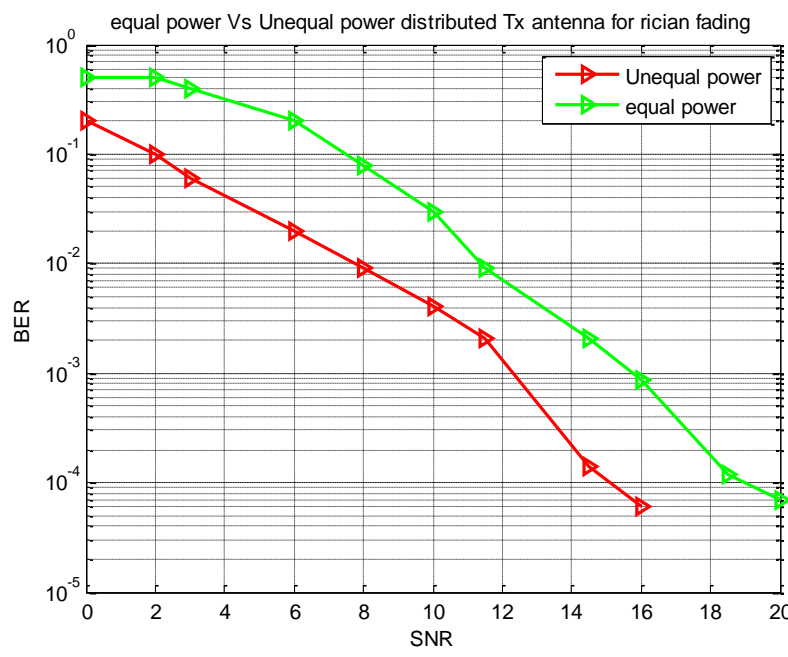

Fig.6 Performance comparison of $2 * 2$ STBC-OFDM with equal and unequal power distributions 
Fig.6 illustrates the results of the proposed approach over rician fading channel with equal an un-equal power distributions. From the fig. 6, it can be noticed that equal power system gives a $3 \mathrm{~dB}$ penalty when compared with unequal power system.

The coherence time was the time above which the value of correlation function is above 0.5 . When the delay constraint of the channel was small compared to coherence time of channel, it is called slow fading channel. Fast fading channel can be defined as the channel at which the channel delay constraint if higher compared with coherence time of channel. The Simulation results for different Doppler frequency $\left(f_{d}\right) 50$, $200,300,500$ are presented in figure.7. Low values of $f_{d}$ denotes slow fading channel and high values denotes fast fading channel.

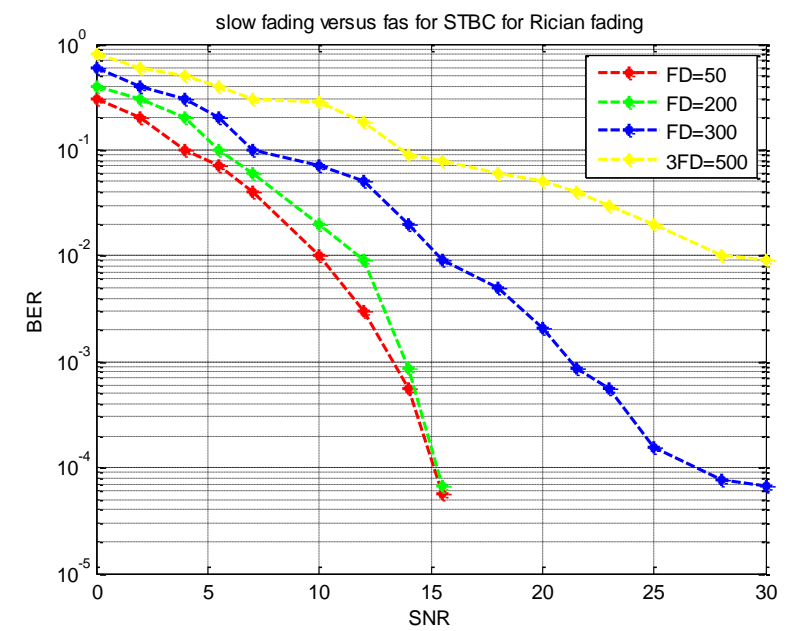

Fig.7. Performance comparison of $2 * 2$ STBC-OFDM with various Doppler frequencies

From the performance result in Figure.7, it can be observed that the performance of STBC-OFDM degrades in fast fading conditions.

\section{CONCLUSIONS}

This paper proposed al Almouti based STBC-OFDM approach for various frequency selective fading channels. This approach didn't require channel state information at receiver or at the transmitter. This approach also used MLE for decoding for recursive decoding. The simulation results were done for two types of channels, Rayleigh and rician fading channels. Simulation results show the performance of the proposed recursive-type receiver to be nearly optimum.

\section{REFERENCES}

[1] V. Tarokh, N. Seshadri, and A. R. Calderbank, "Spacetime codes for high data rate wireless communication: Performance criterion and code construction", IEEE Trans. Inform. Theory, vol.44, no.2, pp.744-765, Mar. 1998.

[2] S. M. Alamouti, "A Simple Transmit Diversity Technique for Wireless Communications", IEEE J. Select. Areas Comm., vol.16, no.8, pp.1451-1458, Oct. 1998.

[3] V. Tarokh, H. Jafarkhani and A. R. Calderbank, "Spacetime block codes from orthogonal designs", IEEE Trans.Inform. Theory, vol.45, no.5, pp. 1456-1467, July 1999.

[4] D. Agrawal, V. Tarokh, A. Naguib and N. Seshadri, "Space-time coded OFDM for high data rate wireless communication over wideband channels", Proc. IEEE VTC'98, pp.2232-2236, 1998.

[5] S. Mudulodu and A. Paulraj, "A transmit diversity scheme for frequency selective fading channels", Proc. IEEE GLOBECOM’ 00, pp.1089-1093, 2000.

[6] Z. Liu, G. B. Giannakis, A. Scaglione and S. Barbarossa, "Decoding and equalization of unknown multipath channels based on block precoding and transmit antenna diversity", Proc. 33rd Asilomar Conf.., pp.1557-1561, 1999.

[7] Y. Li, N. Seshadri and S. Ariyavisitakul, "Channel estimation for OFDM systems with transmitter diversity in mobile wireless channels", IEEE J. Select. Areas Comm., vol.17, no.3, pp.461-471, Mar. 1999.

[8] M. Uysal and C. N. Georghiades, "Efficient implementation of a maximum likelihood detector for space-time coded systems", Proc. Allerton Conf, Oct 2000.

[9] R. Raheli, A. Polydoros and C. Tzou, "Per-survivor processing: A general approach to MLSE in uncertain environments", IEEE Trans. Comm., vol.43, no.2/3/4, Feb./Mar./Apr. 1995, p.354-364.

[10] V. Tarokh, N. Seshadri and A. R. Calderbank, "Spacetime codes for high data rate wireless communication: performance criterion and code construction", IEEE Trans. Inform Theory, vol. 44, $\mathrm{n}^{\circ} 2$, pp. 744-765, Mar. 1998.

[11] S. Baro, G. Bauch and A. Hansmann, "Improved codes for spacetime trellis-coded modulation", IEEE Comm. Letters, vol.4 pp. 20-22, Jan. 2000.

[12] H. Jafarkhani, N. Seshadri, "Super-Orthogonal SpaceTime Trellis Codes", IEEE Trans. Inform. Theory, vol. 49, $\mathrm{n}^{\circ}$ 4, April 2003, pp.937-951.

[13] S. Siwamogsatham and M. P. Fitz, "Improved high rate space-time codes via expanded STBC-MTCM constructions" Proc. IEEE Int. Symp. Information Theory (ISIT), Lausanne, Switzerland, June/July 2002, p.106.

[14] G. Ungerboeck, "Channel coding for multilevel/phase signals", IEEE Trans. Inform. Theory, vol. IT 28, pp. 5567, Jan. 1982

[15] W. Huiming, X. Xiang-Gen, Y. Qinye and L. Bin, "A family of space-time block codes achieving full diversity with linear receivers," IEEE Transactions on Communications, vol. 57, pp. 3607-3617, 2009.

[16] A. Slaney and Y. Sun, "Space-time coding for wireless communications: an overview," IEE Proceedings in Communications, vol. 153, pp. 509-518, 2006.

[17] S. N. Diggavi, N. Al-Dhahir, A. Stamoulis and A. R. Calderbank, "Great expectations: the value of spatial diversity in wireless networks," Proceedings of the IEEE, vol. 92, pp. 219-270, 2004.

[18] D. Agrawal, V. Tarokh, A. Naguib and N. Seshadri, "Space-time coded OFDM for high data-rate wireless communication over wideband channels," in IEEE Conference on Vehicular Technology, vol.3, pp. 2232 2236, 1998.

[19] D. Torrieri and M. C. Valenti, "Efficiently decoded fullrate space-time block codes," IEEE Transactions on Communications, vol. 58, pp. 480-488, 2010. 